\title{
Local and Global Stability Analysis on Flows around a SD7003 Airfoil
}

\author{
Shiwei Qin ${ }^{1}$, Mei Zhuang ${ }^{2}$ \\ Michigan State University, East Lansing, MI 48824 \\ Miguel R. Visbal ${ }^{3}$ \\ U.S Air Force Research laboratory, Wright-Patterson Air Force Base, OH 45433 \\ Marshall C. Galbraith ${ }^{4}$ \\ University of Cincinnati, Cincinnati, $\mathrm{OH} 45221$ \\ Yongsheng Lian ${ }^{5}$ and Wei Shyy ${ }^{6}$ \\ University of Michigan, Ann Arbor, MI 48109
}

\begin{abstract}
Laminar separation and subsequent transition to turbulent flow plays an important role in the performance of airfoils operating at low Reynolds number $\left(\operatorname{Re}<10^{5}\right)$. For better understanding of the formation and turbulent breakdown of laminar separation bubbles (LSB), both local and two-dimensional global linear stability analysis are applied to investigate the stability of a CFD time-averaged non-parallel flowfield over a SD7003 airfoil at Reynolds number $6 \times 10^{4}$. The flow is found to be unstable at all wavenumbers examined. The most unstable modes from local and global stability analysis are close to each other, and the frequency of this mode is in agreement with CFD results. Spatial structures of the disturbances at most unstable modes are discussed. By applying the stability analysis, the temporal flow structures of the LSB can be examined with the most unstable modes of global stability results.
\end{abstract}

\section{Nomenclature}

$\begin{array}{ll}\mathbf{A}, \mathbf{B} & =\text { Assembling mass matrices } \\ C & =\text { Speed of sound } \\ L & =\text { Chord length } \\ M a & =\text { Mach number, } V_{0} / C \\ R e & =\text { Reynolds number, } V_{0} L / v \\ V_{0} & =\text { Freestream reference velocity } \\ f & =\text { Fluid body force (e.g. gravitation) } \\ i & =\text { Imaginary unit } \\ k & =\text { Wavenumber } \\ p & =\text { Pressure } \\ \mathbf{v} & =\text { Velocity vector } \\ \Phi & =\text { Vector of eigenfunctions, }\{\hat{u}, \hat{v}, \hat{w}, \hat{p}\} \\ \rho_{0} & =\text { Reference density }\end{array}$

${ }^{1} \mathrm{PhD}$ Graduate Student, Department of Mechanical Engineering.

${ }^{2}$ Currently, Professor, Department of Aerospace Engineering, The Ohio State University, Associate Fellow AIAA.

${ }^{3}$ Technical Area Leader, Computational Sciences Branch, Aeronautical Sciences Division, Associate Fellow AIAA.

${ }^{4} \mathrm{PhD}$ Graduate Student, Department of Aerospace Engineering \& Engineering Mechanics, Student Member AIAA.

${ }^{5}$ Research Specialist, Department of Aerospace Engineering, Member AIAA.

${ }^{6}$ Professor, Department of Aerospace Engineering, Fellow AIAA. 


$$
\begin{array}{lll}
v & = & \text { Kinematic viscosity } \\
\omega & = & \text { Angular frequency } \\
\nabla^{2} & = & \text { Laplace Operator } \\
\text { Subscripts } & \\
r & = & \text { Real part of complex number } \\
i & = & \text { Imaginary part of complex number } \\
* & = & \text { Two-dimensional operator } \\
\text { Superscripts } & \\
- & = & \text { Base flow quantities } \\
\wedge & = & \text { Amplitude of disturbance quantities } \\
, & = & \text { Disturbance quantities } \\
", " '+ & = & \text { Second- and fourth-order derivatives }
\end{array}
$$

\section{Introduction}

Tn recent years, an interest on small Unmanned Air Vehicles, including Micro Air Vehicles (MAV), capable of 1 performing a wide range of missions has grown from the onset of newly developed micro-system technologies. These vehicles typically operate at the Reynolds number of the order of $10^{4}$ to $10^{5}$ due to the low air speed and the vehicle size.

Under such low Reynolds number conditions, the boundary layer at the onset of the pressure rise may still be laminar, and thus is unable to resist substantial adverse pressure gradients and flow separation. Under certain circumstances, the separated flow experiences laminar-turbulent transition and reattaches to form a laminar separation bubble $(\mathrm{LSB})^{1-3}$. Based on its size, the LSB can be categorized as either a short bubble or a long bubble. A short bubble covers a small portion of the airfoil surface and plays an insignificant factor in modifying the velocity and pressure distributions; a long bubble covers a considerable portion of the surface and significantly changes the inviscid pressure distribution and the peak velocity. The presence of such a bubble, especially a long one, leads to substantial change in the effective airfoil shape, causing the decrease of lift, increase of drag, and consequently, poor power efficiency. The onset and successive breakdown of the LSB at low Reynolds number is know to be detrimental to performance, endurance, and stability of MAV.

As transition is affected by a wide range of parameters including wall roughness, freestream turbulence, pressure gradient, acoustic noise, etc., a comprehensive transition model which accounts for all factors has not been developed. Reynolds-averaged Navier-Stokes (RANS) models $^{4-6}$ and large-eddy simulation (LES) models ${ }^{7}$ are being developed to capture the flow properties and predict the transition. Although much research has been conducted, precise predictions and controls of separation and transition of turbulent flow over the airfoil need further investigation.

Stability analysis is considered to be a promising approach to clarify the mechanism of the onset of oscillatory flow observed in the attached or separated flow over the airfoil ${ }^{8}$. Hammond and Redekopp ${ }^{9}$ used a model separation bubble with the assumption of quasi-parallel flow and performed linear local stability analysis based on onedimensional eigenvalue problems (EVP) of the Orr-Sommerfeld equation to determine the conditions for absolute instability of the LSB. Theofilis et al. ${ }^{10}$, on the other hand, used non-parallel two-dimensional steady flows as base flows in three-dimensional BiGlobal linear stability analyses.

The objective of this study is to apply both local and global stability analyses to study the LSB and the laminarturbulent transition of the flow around a SD7003 airfoil at low Reynolds number. Since a relatively large LSB was observed on the upper surface of the airfoil for a range of low Reynolds numbers, the flow around a SD7003 has been extensively studied by both experiments and computations. In the current stability study, the base flows for various angles of attack will be provided by RANS and LES methods ${ }^{7,11}$. Results of local and global stability analyses will be compared to CFD results, and stability features are discussed.

\section{Mathematical Formulations}

\subsection{Global Stability Formulations}

The analysis is based on numerical solution of the two-dimensional partial-derivative eigenvalue problem, which describes small-amplitude three-dimensional disturbances developing on a two-dimensional steady or time-averaged base flow around an airfoil. The base flow is assumed to be infinitely long in the spanwise direction denoted by $\mathrm{z}$ 
such that $\partial_{z}=0$ in the base flow. Slight compressibility is also assumed to eliminate the singularity in the eigenproblem. The nondimensional scales for length, velocity, time, density, and kinematic pressure are taken to be $L, V_{o}, L / V_{0}, \rho_{0}$, and $C V_{0}$, where $C$ is the acoustic speed of the fluid. The dimensionless form of the continuity equation is

$$
\frac{D \rho}{D t}+\rho \nabla \cdot \mathbf{V}=0
$$

With slight compressibility, given the pressure is only a function of density for kinematic pressure $p$,

$$
\frac{D p}{D t}=\frac{1}{\rho M a} \frac{D \rho}{D t}
$$

where $M a=V_{0} / C$ is the Mach number of the flow. Substituting Eq. (2) into Eq. (1), a modified continuity equation can be written as

$$
\frac{D p}{D t}+\frac{1}{M a} \nabla \cdot \mathbf{V}=0
$$

Under slight compressibility conditions, considering the kinematic viscosity $v$ of fluid constant and $f$ a constant body force (e.g. gravitation), the nondimensional momentum equation can be written as

$$
\frac{D \mathbf{v}}{D t}=-\frac{1}{M a} \nabla p+\frac{1}{R e}\left[\nabla^{2} \mathbf{v}+\frac{1}{3} \nabla(\nabla \cdot \mathbf{v})\right]+f
$$

where $R e=V_{0} L / v$ is the Reynolds number.

\subsubsection{Base Flow}

Assumed to be infinitely long in the spanwise direction, the base flow is two-dimensional and steady with the simplified equations

$$
\begin{gathered}
\nabla \cdot \overline{\mathbf{V}}=0 \\
\overline{\mathbf{V}} \cdot \nabla_{*} \overline{\mathbf{V}}=-\nabla_{*} \bar{P}+\frac{1}{R e} \nabla_{*}^{2} \overline{\mathbf{V}}
\end{gathered}
$$

where $\overline{\mathbf{V}}$ and $\bar{P}$ are the velocity and kinematic pressure in the base flow, respectively. $\nabla_{*}$ represents the twodimensional gradient operator.

\subsubsection{Perturbation Equations}

Any flow quantity may be decomposed into a steady base component and an unsteady perturbation component in the following form.

$$
\mathbf{v}(x, y, z, t)=\overline{\mathbf{V}}(x, y)+\mathbf{v}^{\prime}(x, y, z, t), p(x, y, z, t)=\bar{P}(x, y)+p^{\prime}(x, y, z, t)
$$

while the perturbations $u^{\prime}, v^{\prime}, w^{\prime}, p^{\prime}$ have a harmonic dependence on the homogeneous spatial coordinate $\mathrm{z}$ and time,

$$
p^{\prime}=i \hat{p}(x, y) e^{i k z+\omega t}
$$




$$
\begin{aligned}
& u^{\prime}=i \hat{u}(x, y) e^{i k z+\omega t} \\
& v^{\prime}=i \hat{v}(x, y) e^{i k z+\omega t} \\
& w^{\prime}=\hat{w}(x, y) e^{i k z+\omega t}
\end{aligned}
$$

In Eqs. (8) to (11), $i$ is the imaginary unit, $k$ is the spanwise wavenumber, and $\omega=\omega_{r}+i \omega_{i}$ denotes the complex growth rate. The reason for the imaginary amplitude in the normal modes is to avoid complex arithmetic in calculation of the eigenproblem.

Substituting Eq. (7) into the continuity Eq. (3) and the momentum Eq. (4), subtracting the base flow Eqs. (5) and (6), neglecting the high order terms of perturbations and replacing the perturbations with Eqs. (8) to (11) result in an eigenproblem of perturbations with a growth rate as the eigenvalue and perturbation amplitudes $\hat{u}, \hat{v}, \hat{w}, \hat{p}$ as the eigenfunctions.

$$
\begin{gathered}
\omega \hat{u}+\left(\overline{\mathbf{V}} \cdot \nabla_{*}\right) \hat{u}+\left(\hat{\mathbf{v}} \cdot \nabla_{*}\right) \bar{U}+M a^{-1} \frac{\partial \hat{p}}{\partial x}=R^{-1}\left[\left(\nabla_{*}^{2}-k^{2}\right) \hat{u}+\frac{1}{3} \frac{\partial}{\partial x}\left(\nabla_{*} \cdot \hat{\mathbf{v}}+k \hat{w}\right)\right] \\
\omega \hat{v}+\left(\overline{\mathbf{V}} \cdot \nabla_{*}\right) \hat{v}+\left(\hat{\mathbf{v}} \cdot \nabla_{*}\right) \bar{V}+M a^{-1} \frac{\partial \hat{p}}{\partial y}=\operatorname{Re}^{-1}\left[\left(\nabla_{*}^{2}-k^{2}\right) \hat{v}+\frac{1}{3} \frac{\partial}{\partial y}\left(\nabla_{*} \cdot \hat{\mathbf{v}}+k \hat{w}\right)\right] \\
\omega \hat{w}+\left(\overline{\mathbf{V}} \cdot \nabla_{*}\right) \hat{w}-M a^{-1} k \hat{p}=\operatorname{Re}^{-1}\left[\left(\nabla_{*}^{2}-k^{2}\right) \hat{w}-\frac{1}{3} k\left(\nabla_{*} \cdot \hat{\mathbf{v}}+k \hat{w}\right)\right] \\
\omega \hat{p}+\left(\overline{\mathbf{V}} \cdot \nabla_{*}\right) \hat{p}+\left(\hat{\mathbf{v}} \cdot \nabla_{*}\right) \bar{P}+M a^{-1}\left(\nabla_{*} \cdot \hat{\mathbf{v}}+k \hat{w}\right)=0
\end{gathered}
$$

For stability analysis around the separated flow, the following boundary conditions are implemented: homogeneous Dirichlet conditions are imposed on the disturbance velocity components at the solid wall and all disturbance components in the free-stream. On the solid wall, the compatibility conditions (Eqs. (16) and (17)) are derived from the governing equations for the pressure perturbation. The same homogeneous Dirichlet conditions apply to all disturbances components at the inflow boundary, which, from a physical point of view, serves to ensure that no perturbations other than potentially self-excited global eigen-modes enter the separated flow region. Linear extrapolation from the interior of the domain is applied for the disturbances on the outflow boundary.

$$
\left(-\frac{1}{M a} \hat{p} \mathbf{I}^{*}+\frac{1}{R e}\left(\nabla \hat{\mathbf{v}}+\frac{1}{3} I^{*}\left(\nabla_{*} \cdot \hat{\mathbf{v}}+k \hat{w}\right)\right)\right) \cdot \mathbf{n}=0
$$

$$
\nabla \hat{w} \cdot \mathbf{n}=0
$$

Finite element method for the discretization of Eqs. (12)-(15) and imposition of the boundary conditions results in a real non-symmetric generalized eigenvalue problem in the form

$$
\mathbf{A} \Phi=\omega \mathbf{B} \Phi
$$

where $\mathbf{A}$ and $\mathbf{B}$ are the assembling mass matrices calculated from the base flow and $\Phi=\{\hat{u}, \hat{v}, \hat{w}, \hat{p}\}$ is the assembling vector of the eigenfunctions. According to linear stability theory, the stability properties of the flow depend on the eigenvalue $\omega$. If $\omega$ is real, the disturbances either grow or decay monotonically, the critical Reynolds number is one in which $\omega=0$. If $\omega$ is complex, the neutral condition is $\omega_{r}=0$, and the onset of instability is oscillatory with dimensionless angular frequency $\omega_{i}$. This normal mode form also includes the timedependent two-dimensional instability of the steady flow for which $k=0$. 
With eigen-modes from stability computation, the total three-dimensional flow field can be reconstructed on the basis of the two-dimensional base flow with an arbitrary number of disturbance eigen-modes. When these eigenmodes are chosen to be conjugate pairs of most unstable modes of an eigenspectrum, the real three-dimensional flows can be expressed by the following superposition of eigenfunctions (the pressure and velocity $v$ have a similar form as that of $u$ )

$$
\begin{gathered}
u=\bar{U}-2 \sum_{n=1}^{N} e^{\omega_{r}^{n} t} \cos (k z)\left[\hat{u}_{i}^{n} \cos \left(\omega_{i}^{n} t\right)+\hat{u}_{r}^{n} \sin \left(\omega_{i}^{n} t\right)\right] \\
w=2 \sum_{n=1}^{N} e^{\omega_{r}^{n} t} \cos (k z)\left[\hat{w}_{r}^{n} \cos \left(\omega_{i}^{n} t\right)-\hat{w}_{i}^{n} \sin \left(\omega_{i}^{n} t\right)\right]
\end{gathered}
$$

where subscripts $r, i, n, N$ denotes the real and imaginary parts of the eigenvalues or eigenfunctions, the $n_{t h}$ pair of eigen-modes and total number of conjugate pairs of eigen-modes, respectively.

\subsection{Local Stability Formulations}

Local stability analysis is also carried out for the same base flow, and its results are compared to those of global stability analysis, as the first validation step of global stability computations. The base flow closely satisfies $\partial x<<\partial y$ at all locations, therefore it is reasonable to assume that, in a short streamwise distance, the flow is a parallel flow, which justifies the application of local stability analysis to local x-direction velocity profiles $\bar{U}$ at different streamwise locations.

For a parallel viscous flow $\bar{U}(y)$, with perturbations assumed in form of Eq. (21), the temporal local stability properties are governed by the Orr-Sommerfeld relation ${ }^{12}$, Eq. (22). For simplification, the viscosity is neglected and the Orr-Sommerfeld equation is reduced to the Rayleigh equation ${ }^{12}$ (23) with boundary conditions, Eq. (24).

$$
\begin{gathered}
\left(u^{\prime}, v^{\prime}, w^{\prime}, p^{\prime}\right)=[\hat{u}(y), \hat{v}(y), \hat{w}(y), \hat{p}(y)] \exp (i k x+\omega t) \\
\left(\bar{U}-i \frac{\omega}{k}\right)\left(\hat{v}^{\prime \prime}-k^{2} \hat{v}\right)-\bar{U}^{\prime \prime} \hat{v}+\frac{i v}{k}\left(\hat{v}^{\prime \prime \prime}-2 k^{2} \hat{v}^{\prime \prime}+k^{4} \hat{v}\right)=0 \\
\hat{v}^{\prime \prime}-\left(k^{2}+\frac{\bar{U}^{\prime \prime}}{\bar{U}-i \omega / k}\right) \hat{v}=0 \\
\hat{v}(0)=0 \text { and } \hat{v}(y \rightarrow \infty) \sim e^{-k y}
\end{gathered}
$$

A smoothing-spline fitting method is developed in the current study which enables exact fitting of any base flow profile from experiments or computations with smooth first- and second-order derivatives. From previous study by other researchers ${ }^{13-15}$, analytical expressions of velocity profiles were used to perform local stability analysis. Unfortunately, analytical profile expressions for data from experiments or CFD are not practical. Inaccurate fitting of a base flow could cause significant error in stability analysis due to the fact that stability analysis is very sensitive to base flows. The smoothing-spline fitting method gives a nearly perfect fitting in the current study. The method is directly coupled with the eigensolver which eliminates the necessity of analytical expressions of the base flow velocity and its derivatives. The shooting method is used in the eigensolver ${ }^{16}$.

\section{Base Flow}

The base flow used in the present work is provided by Galbraith and Visbla ${ }^{7}$. A high-order implicit large-eddy simulation (ILES) solver is used in their computation. The base flow is the time-averaged result for the flow around a SD7003 airfoil at Reynolds number $6 \times 10^{4}$ with angle of attack of $4^{\circ}$. The computation domain (shown in Fig. 1 enclosed by the rectangle) used for global stability analysis is cut in the range $\mathrm{x} / \mathrm{C} \in[0.1941,1.0156]$ and $\mathrm{y} / \mathrm{C} \in$ [0.0011, 0.2914], which ensures that the whole LSB is enclosed. 


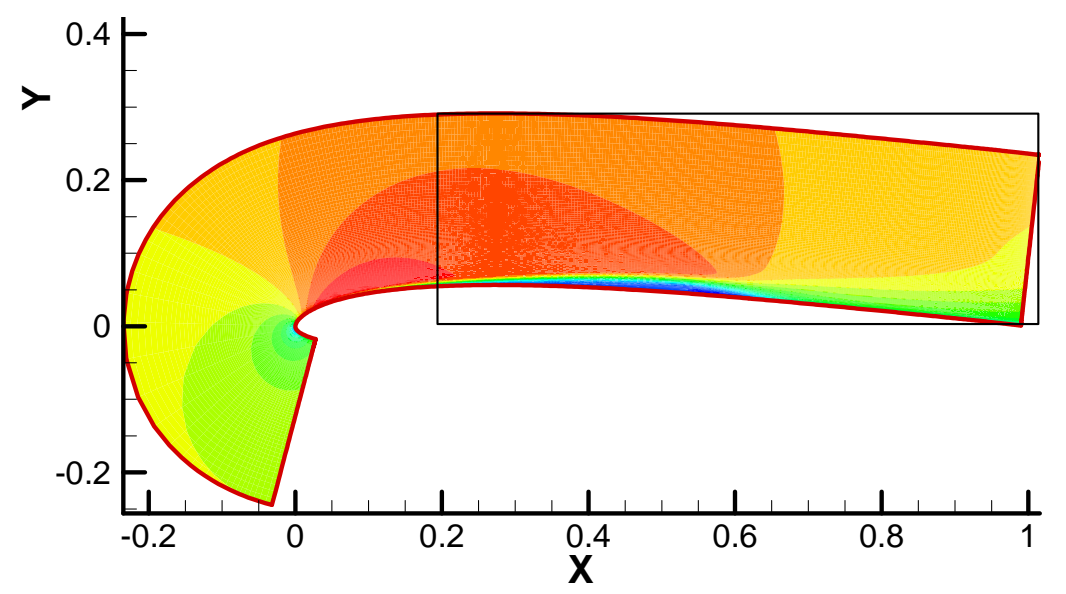

Figure 1. Base flow around a SD7003 airfoil, $R e=6 \times 10^{4}$, Angle of attack $=$ $4^{\circ}$, Galbraith and $\operatorname{Visbal}^{7}$ (LES model, colored by $\bar{U}$ velocity)

\section{Local Stability Results}

To detect the most unstable mode, one dimensional stability analysis is applied to the parallel $\mathrm{x}$-direction base flow velocity profile at $\mathrm{x} / \mathrm{C}=0.5$ which is shown in Fig. 2 . The reason for choosing this location is that $\mathrm{x} / \mathrm{C}=0.5$ is about the center of the LSB where maximum disturbances are assumed to occur. The Rayleigh Eq. (23) with boundary condition Eq. (24) is solved for the eigen-modes. Details of the numerical procedures can be found in Refs. 15. Figure 3 shows the most unstable mode of $\omega=18.57+42.11$, which is close to the most unstable mode obtained from global stability analysis which is elaborated in the following section. Considering that this mode comes from only one single profile at location $\mathrm{x} / \mathrm{C}=0.5$ and that only $\mathrm{x}$-direction velocity is taken for the analysis, the two most unstable modes from local and global stability analysis are in good agreement. Unless otherwise stated, all quantities in results are dimensionless.

\section{Global Stability Results}

Our program code based on the formulations in section II is compiled to carry out the computation of global stability analysis. To validate the code, a series of computations on the global stability of lid-driven cavity flow, which has the same formulation but different base flow and boundary conditions, were performed. The results are in agreement with those obtained by Ding $^{17}$ and Theofilis ${ }^{18}$, which therefore indicates the reliability of our computation.

Mesh convergence test was performed on meshes with the following resolutions: $29 \times 21,41 \times 30$, $47 \times 35, \quad 56 \times 41, \quad 70 \times 51$, and $76 \times 80$, shown in Fig. 4. The greatest difference between meshes $(70 \times 51$ and $76 \times 80)$ is about $3 \%$, which indicates that mesh $(70 \times 51)$ sufficiently computes accurate leading eigenvalues. This non-uniform mesh $(70 \times 51)$ is chosen for the following computation cases.

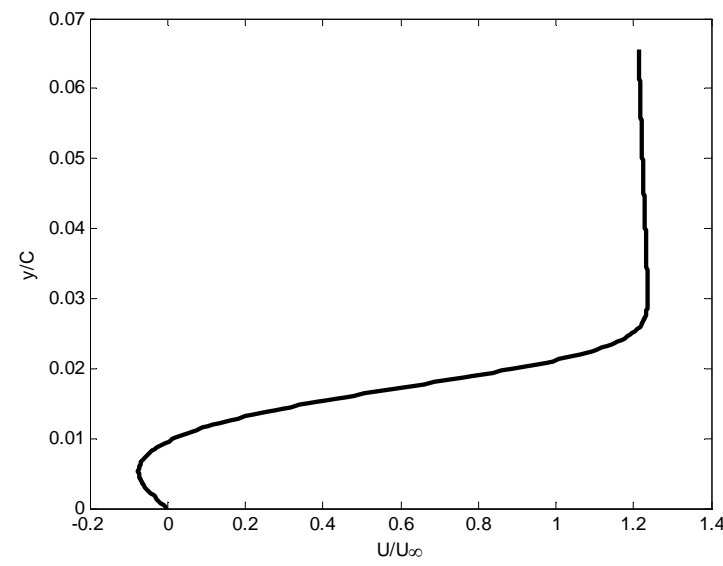

Figure 2. Time-averaged $\mathbf{x}$ direction velocity profile $\bar{U}$ at $\mathbf{x} / \mathbf{C}=\mathbf{0 . 5}$ 


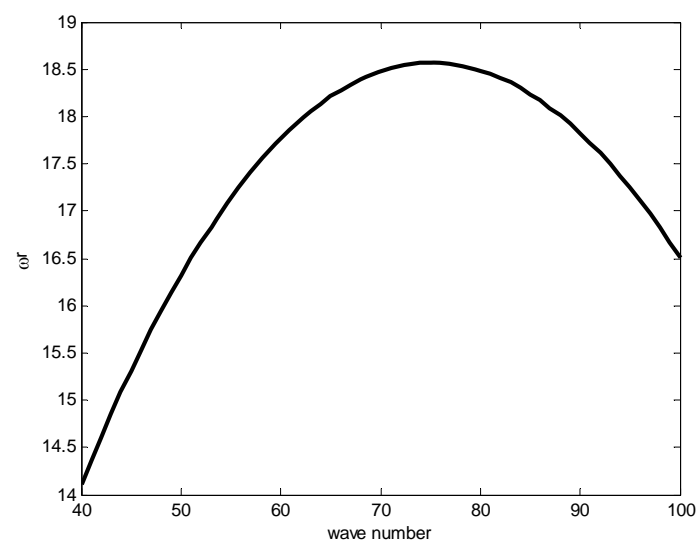

a)

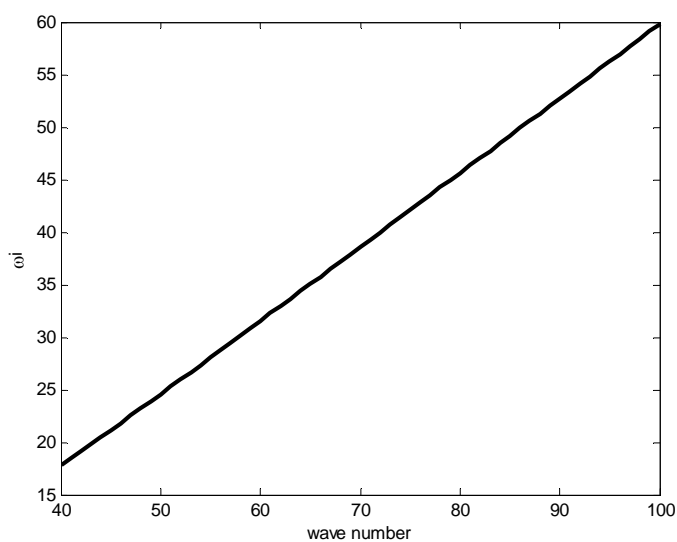

b)

Figure 3. Local stability analysis for time-averaged $\mathbf{x}$-direction velocity profile $\bar{U}$ at $\mathbf{x} / \mathbf{C}=\mathbf{0 . 5}$. a) growth rate vs. wavenumber, b) angular frequency vs. wavenumber.

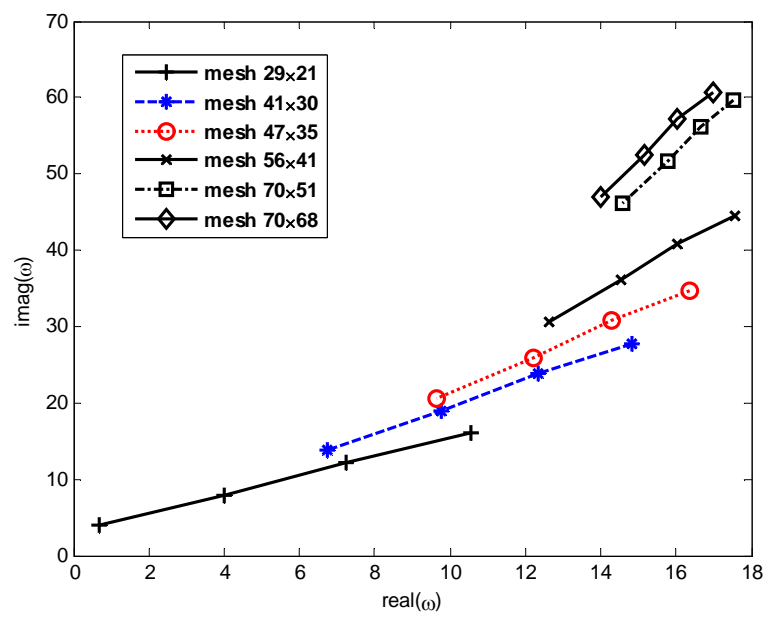

Figure 4. The four most unstable modes at wavenumber $k=20$ with different base flow meshes.

A range of wavenumbers were examined with parameter $\mathrm{k} \in[0.2,90]$, which corresponds to the periodic spanwise extent of the domain $\mathrm{Lz} / \mathrm{Lx} \in[0.085,38]$, where $\mathrm{Lz}$ is the disturbance wavelength and $\mathrm{Lx}=0.8215$ is the dimensionless streamwise extent of the separated region monitored. The dependence of the most unstable eigenvalue (the one with largest real part) on the wavenumber is plotted in Fig. 5, which shows an unstable flow for the whole range of wavenumbers examined. Moreover, the growth rate of the most unstable mode is a monotonically decreasing function of the wavenumber, so increasing angular frequency with increasing wavenumber means shorter wavelength perturbations are less unstable. With wavenumber $\mathrm{k}$ close to zero, the most unstable mode reaches a plateau of $\omega=18+59.4 \mathrm{i}$ of which the angular frequency 59.4 corresponds to a frequency of 9.4. Galbraith and Visual ${ }^{7}$ monitored the velocity history at a point directly above the airfoil surface at $\mathrm{x} / \mathrm{C}=0.5$, about the center of the LSB, and plotted the spectrum of turbulent kinetic energy which is cited as Fig. 6. Galbraith and Visual pointed out that there is a broad peak centered at frequency 9.0, and this is verified by the most unstable mode frequency in current global stability computation, which suggests that global stability analysis is a promising approach for study of oscillatory phenomena observed in CFD results. 

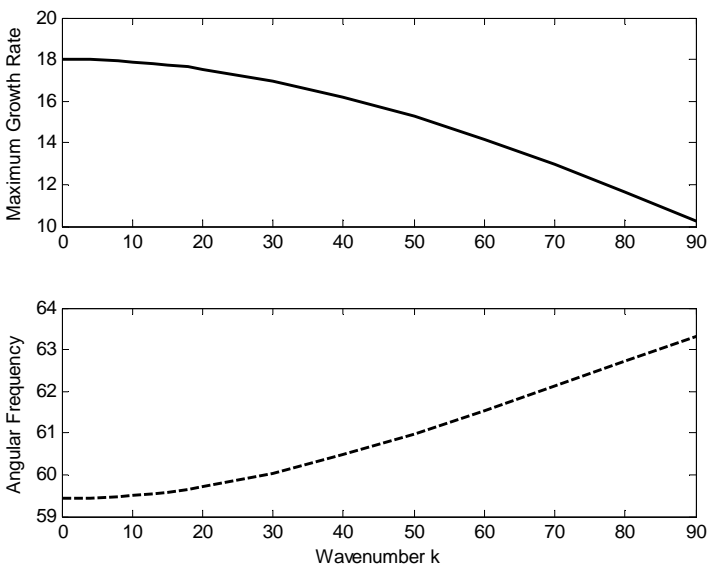

Figure 5. Most unstable mode vs. wavenumber: (upper) maximum growth rate, (lower) angular frequency.

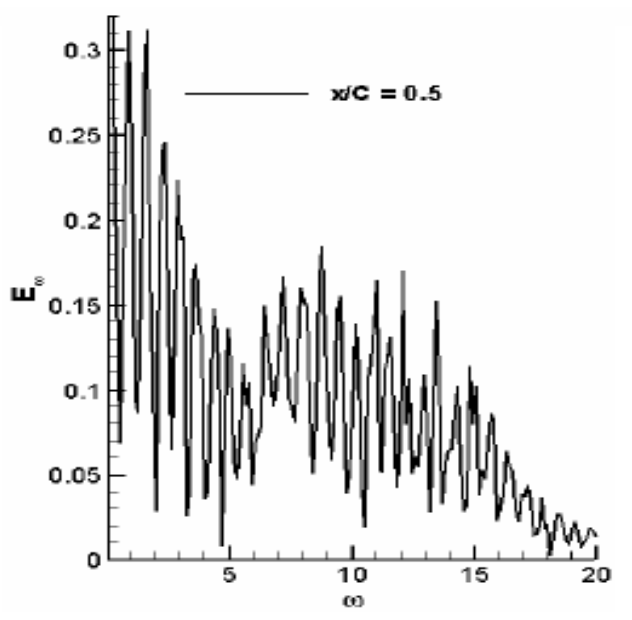

Figure 6. Turbulent-kinetic-energy frequency spectra at $\mathbf{x} / \mathrm{C}=0.5$ (center of LSB), cited from Ref. 7

According to Eqs. (19) and (20), the three-dimensional total velocity field can be reconstructed by adding the summation of a number of eigen-modes to the base flow. Figure 7 shows the spatial distribution of total $\mathrm{x}$-direction velocity on the Oxy plane with the conjugate pair of most unstable eigen-modes for $\mathrm{Lz} / \mathrm{Lx}=7.6484(\mathrm{k}=1)$. The eigenvalue for the most unstable mode is $\omega=18+59.4 \mathrm{i}$ with a dimensionless period $\mathrm{T}=0.1058$. Figures. $7 \mathrm{a}-7 \mathrm{~d}$ correspond to instant times $0, \mathrm{~T} / 4, \mathrm{~T} / 2,3 \mathrm{~T} / 4$, respectively. Since the growth rate of the most unstable mode is greater than zero, the disturbances will amplify as time elapses and become dominant over the base flow. Figures $7 \mathrm{c}$ and $7 \mathrm{~d}$ can be considered the spatial distribution of only the x-direction velocity disturbance while the base flow $\bar{U}$ still has obvious effect on the shape of velocity contours in Figs. 7a and 7b. It should be noted that the qualitative structure of the most unstable eigen-mode is almost independent of wavenumber $\mathrm{k}$ such that the following results can be considered representative of all those obtained at different wavenumber values. As shown in Figs. 7-9, all three velocity disturbances share some similar properties. First, disturbances are zero at inlet, airfoil surface and farfield boundaries, as imposed by the boundary conditions. It should be noted that zero disturbances also occur at the outlet, which is not restricted by the boundary condition. Second, all disturbances are confined in the region $0.2<$ $\mathrm{x} / \mathrm{C}<0.65$ and $\mathrm{y} / \mathrm{C}<0.2$, which corresponds to the LSB region where reverse flow occurs in the base flow. This can be explained by the fact that stability is closely related to reverse flow. Third, both maximum magnitude and maximum gradient of disturbances occur at location around $\mathrm{x} / \mathrm{C}=0.4$, the center of the LSB, therefore it is reasonable to say that the LSB center is most likely to amplify an infinitesimal disturbance and thus is the most unstable point of the flow.

Comparisons of Fig. 7c, Fig. 8 and Fig. 9, which are representative of x-, y-and z-direction velocity disturbances, respectively, show that the magnitude of $z$-direction disturbance is much weaker (close to $O\left(10^{-2}\right)$ ) than those of $x$ and y-direction disturbances. This difference in disturbance magnitude can be shown clearly by comparing the magnitudes of their eigenfunctions. Similar difference in disturbances is also found in Ding's results ${ }^{17}$. The spanwise eigen-modes indicate the existence of oscillatory flow structure, though weak compared to the other two directions, perpendicular to the base flow plane. More investigation is needed to show this phenomenon in numerical simulations and experimental observations.

A special note is given to the spatial structure in the distribution of the disturbances clearly shown in Figs. 7-9. For each figure, the LSB region is divided into several neighboring regions (cells) in terms of the arithmetic value of disturbances, and the locations of cells are similar in all three disturbances. In each two neighboring cells at a time instant, one has a disturbance velocity with direction opposite to the other cell while both are similar in absolute magnitude. Similar structures are also found by Theofilis ${ }^{19,20}$. It can be conjectured that this cellular structure can reveal the vortex configuration inside of the LSB, which requires further investigation of the stability results and verification from numerical and experimental results. 


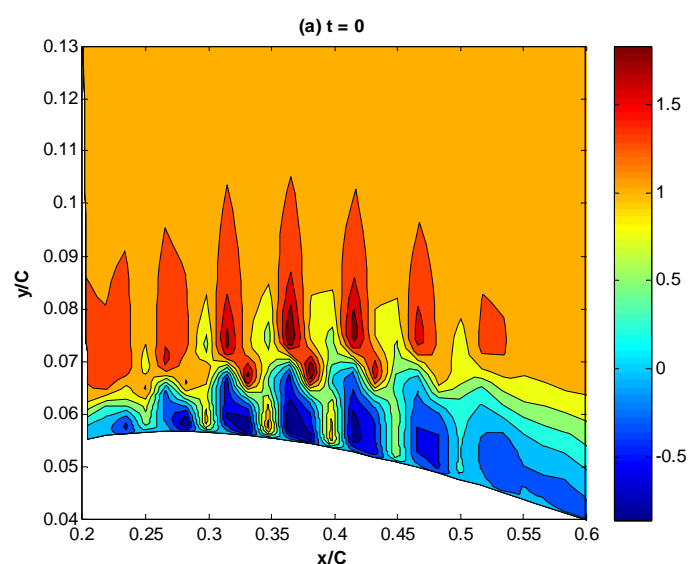

a)

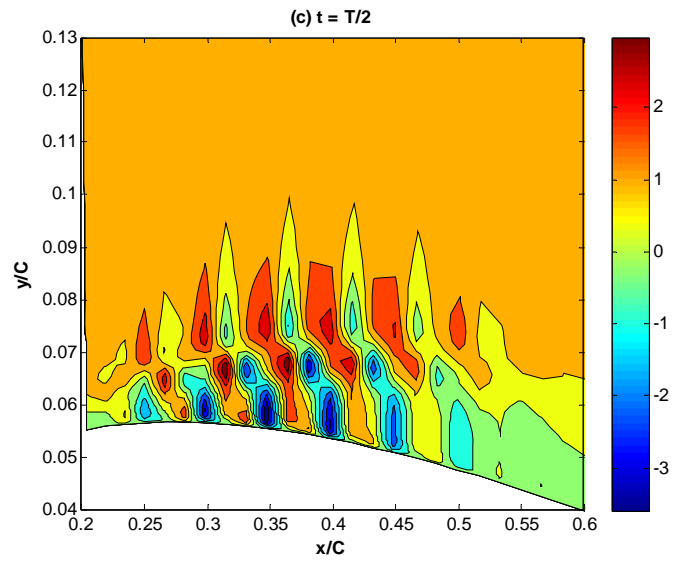

c)

Figure 7. Total $x$-direction velocity with the conjugate pair of most unstable modes for $\mathbf{L z} / \mathbf{L x}=7.6484(\mathrm{k}$

= 1). 7a) $\mathrm{t}=0,7 \mathrm{~b}) \mathrm{t}=\mathrm{T} / 4,7 \mathrm{c}) \mathrm{t}=\mathrm{T} / 2,7 \mathrm{~d}) \mathrm{t}=3 \mathrm{~T} / 4$

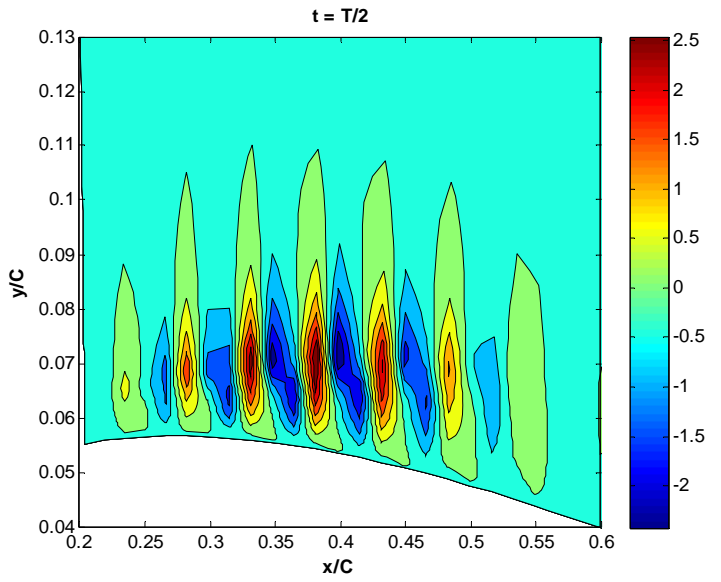

Figure 8. y-direction velocity disturbance with the conjugate pair of most unstable modes for $\mathrm{Lz} / \mathrm{Lx}=$ $7.6484(k=1)$ at $t=T / 2$

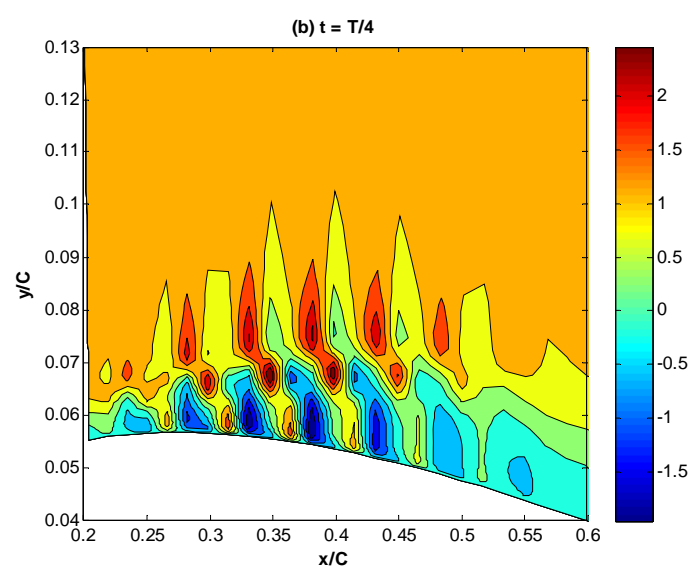

b)

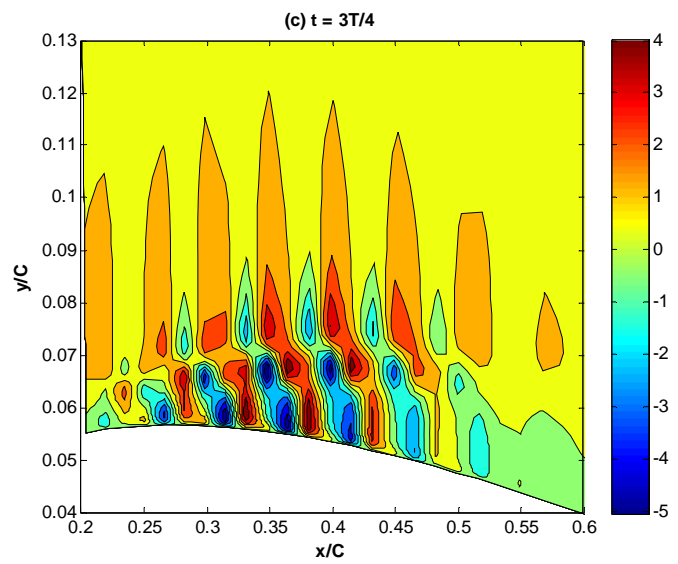

d)

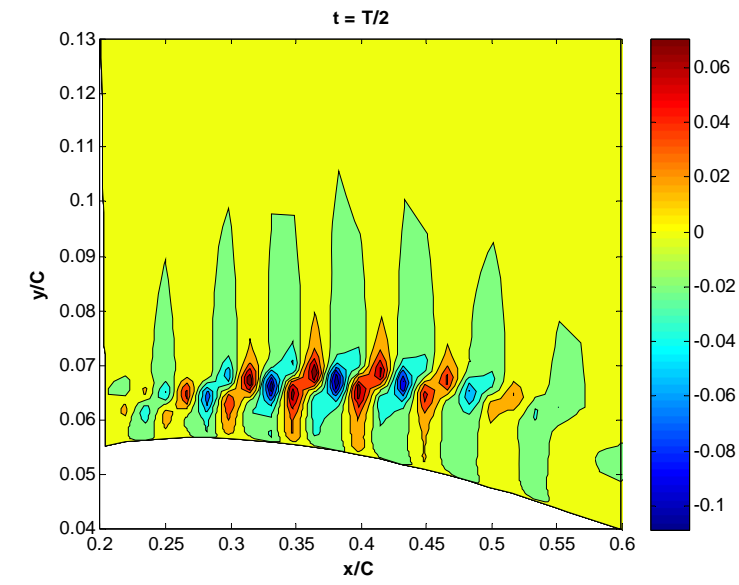

Figure 9. z-direction velocity disturbance with the conjugate pair of most unstable modes for $\mathrm{Lz} / \mathrm{Lx}=7.6484(\mathrm{k}=1)$ at $\mathrm{t}=\mathrm{T} / 2$ 


\section{Conclusion}

The LSB region of a time-averaged flow field around a SD7003 airfoil at Reynolds number $6 \times 10^{4}$ with angle of attack $4^{\circ}$ is studied using both local and global stability analysis. The base flow is obtained using a compressible solver based on implicit large eddy simulation (ILES) method. The partial derivative eigenvalue problem is solved in global stability analysis while the classic Rayleigh equation is solved for local stability.

While the most unstable frequencies from both methods are in close agreement, global stability analysis results are closer to CFD results and reveal more features of the flow. Stability analysis shows the flow is unstable at this Reynolds number and will transit to turbulent flow with introduction of disturbances, which is verified by the CFD results. Development of flow structure and disturbance distribution is discussed, and it is found that the disturbances are confined in the LSB region with maximum magnitude and maximum gradient in the center of the bubble. Cellular structure of the disturbance distribution is specially noted while more numerical and experimental data are needed to verify its relation to the vortex configuration inside of the LSB.

Future work will investigate the effects of the Reynolds number and angle of attack on the stability features of LSB, and relate stability analysis results to flow features found numerically and experimentally, with focus on possibly helpful information concerning flow control.

\section{Acknowledgement}

This research has been supported by the U.S Air Force Research Laboratory at Wright-Patterson Air Force Base through subcontract \# 3000588512 from the University of Michigan.

\section{References}

1'Jones, B. M., "Stalling," Journal of the Royal Aeronautical Society, Vol. 38, 1938, pp. 747-770.

${ }^{2}$ Young, A. D., and Horton, H. P., "Some Results of Investigation of Separation Bubbles," AGARD Conference Proceedings, Vol. 4, AGARD, London, 1966, pp. 779-811.

${ }^{3}$ Tani, I., "Low Speed Flows Involving Bubble Separations," Progress in Aeronautical Sciences, Pergamon, New York, 1964, pp. $70-103$.

${ }^{4}$ Radespiel, R., Graage, K., and Brodersen, O., "Transition Predictions Using Reynolds-Averaged Navier-Stokes and Linear Stability Analysis Methods," AIAA Paper 91-1641, June 1991.

${ }^{5}$ Stock, H. W., and Haase, W., "A Feasibility Study of eN Transition Prediction in Navier-Stokes Methods for Airfoils," AIAA Journal, Vol. 37, No. 10, 1999, pp. 1187-1196.

${ }^{6}$ Yuan, W., Khalid, M., Windte, J., Scholz, U., and Radespiel, R., “An Investigation of low Reynolds-Number Flows Past Airfoils," 23rd AIAA Applied Aerodynamics Conference, Toronto, AIAA Paper 2005-4607.

${ }^{7}$ Marshall C. Galbraith, and Miguel R. Visbal, "Implicit large Eddy Simulation of Low Reynolds Number Flow Past the SD7003 Airfoil," AIAA Paper 2008-225.

${ }^{8}$ Asei Tezuka, "Global stability Analysis of Attached or Separated Flows over a NACA0012 Airfoil," AIAA Paper 20061300.

${ }^{9}$ Hammond, D. A. and Redekopp, "Local and Global Instability Properties of Separation Bubbles,” Eur. J. Mech. B/Fluids, Vol. 2, 1998, pp. 145-164.

${ }^{10}$ Theofilis, V., Hein, S. and Dallmann, U., "On the Origins of Unsteadiness and Three-Dimensionality in a Laminar Separation Bubble," Phil. Trans. Roy. Soc. Lond. A. Vol. 358, 2000, pp. 3229-3246.

${ }^{11}$ Lian Y., and Shyy W., "Laminar-turbulent Transition of a Low Reynolds Number Rigid or Flexible Airfoil," AIAA Journal, vol. 45, No. 7, 2007.

${ }^{12}$ Frank M. White, "Viscous Fluid Flow (Third Edition)," McGraw-Hill, NY, 2006.

${ }^{13}$ Huerre P., and Monkewitz P. A., "Absolute and Convective Instability in Free Shear Layers," J. Fluid Mech, vol 159, 1985, pp. 151-168.

${ }^{14}$ Triantafyllou G. S., Triantafyllou M. S., and Chryssostomidis C., "On the formation of vortex streets behind stationary cylinders," J. Fluid Mech. Vol. 170, 1986, pp. 461-477.

${ }^{15}$ Zhuang, M., Dimotakis, P. E. and Kubota, T. [1990], "The Effect of Walls on a Spatial Growing Supersonic Shear Layer," Physics of Fluids, 2(4), 599-604.

${ }^{16}$ Zhuang, M., Kubota, T. and Dimotakis, P. E. "On the instability of inviscid, compressible free shear layers," AIAA Journal, Vol. 28, No.10, 1990, pp. 1728-1733.

${ }^{17}$ Ding Y, and Kawahara M., "Linear Stability of Impressible Flow Using a Mixed Finite Element Method,” J. Comp. Phys, Vol. 139, 1998, pp. 243-273.

${ }^{18}$ Theofilis, V., Duck, P., and Owen, J., "Viscous Linear Stability Analysis of Rectangular Duct and Cavity Flows", J. Fluid Mech. Vol. 505, 2004, pp. 249-268.

${ }^{19}$ Theofilis, V. and Sherwin, S. J., "Global Instabilities in Trailing-Edge Laminar Separated Flow on a NACA 0012 Airfoil", International Symposium on Airbreathing Engines XV, ISABE 2001-1094, Bangalore, India, 2001. 
${ }^{20}$ Theofilis, V., Barkley, D. and Sherwin, S. J., "Spectral/hp Element Technology for Flow Instability and Control", Aeronautical Journal, Vol. 106, 2002, pp. 619-625. 